Baltic Astronomy, vol. 6, 335, 1997.

\title{
THE GENERAL CATALOG OF POSITIONS AND PROPER MOTIONS OF 6637 BRIGHT STARS (NEWBS)
}

E. V. Khrutskaya

Pulkovo Observatory, Russian Academy of Sciences, St. Petersburg 196140, Russia

Received July 15, 1996.

Key words: astrometry - stars: catalogs

New positions and proper motions for stars to a limiting magnitude of 7.9 over the whole sky (NewBS) were obtained as a result of a thorough revision of observational material of the International Bright Star Program. 21 catalogs with right ascensions $(\alpha)$ and 16 catalogs with declinations $(\delta)$ were used to derive new positions for bright stars. The mean epoch of observation for positions in NewBS is 1968.4. The mean rms errors of positions in NewBS for the mean epoch of observation are: $\pm 0.003 \sec \delta$ for $\alpha$ and $\pm 0 . " 08$ for $\delta$. The errors of new centennial proper motions are $\pm 0.012 \sec \delta$ and $\pm 0 . " 19$ for $\alpha$ and $\delta$, respectively. All stars listed in the NewBS catalog will be incorporated into the HIPPARCOS catalog.

We present the results of a comparison of NewBS data with those of FK5 Extension, CAMC-4, CGS and BS. External errors of the NewBS catalog with respect to FK5 catalog for the epoch of 2000.0 are: $\pm 0.005 \sec \delta$ and $\pm 0.013 \sec \delta$ for $\alpha$ and $\mu_{\alpha}$ and $\pm 0 . " 11$ and $\pm 0 . " 22$ for $\delta$ and $\mu_{\delta}$, respectively. On the whole, smoothed systematic differences (NewBS-FK5) for both coordinates lie within $\pm 0 . " 02$. The most important (NewBS-CAMC-4) systematic differences for both coordinates are found to be dependent on declination. For right ascensions the greatest differences are found north of $+75^{\circ}$. The greatest declination differences are found within the declination zone from $-30^{\circ}$ down to $-42.5^{\circ}$. These differences agree well with the (FK5-CAMC-4) systematic differences. 